\title{
Immunohistochemical Localization of Anoctamin 1 in the Mouse Cerebellum
}

\author{
Yong Soo Park ${ }^{1,2}$, Ji Hyun Jeon ${ }^{1}$, Seung Hee Lee ${ }^{1,2}$, Sun Sook Paik ${ }^{1,2}$, In-Beom Kim ${ }^{1,2,3 *}$ \\ ${ }^{1}$ Department of Anatomy, College of Medicine, The Catholic University of Korea, Seoul 06591, Korea \\ ${ }^{2}$ Catholic Neuroscience Institute, College of Medicine, The Catholic University of Korea, Seoul 06591, Korea \\ ${ }^{3}$ Catholic Institute for Applied Anatomy, College of Medicine, The Catholic University of Korea, Seoul 06591, Korea
}

*Correspondence to:

Kim IB,

(iD http://orcid.org/0000-0002-1932-8407

Tel: $+82-2-2258-7263$

Fax: +82-2-536-3110

E-mail: ibkimmd@catholic.ac.kr

Received December 3, 2018

Revised December 19, 2018

Accepted December 19, 2018
Since a transmembrane protein, TMEM16A, also called anoctamin 1 (ANO1), was identified as a bona fide calcium $\left(\mathrm{Ca}^{2+}\right)$-activated chloride $\left(\mathrm{Cl}^{-}\right)$channel $(\mathrm{CaCC})$, there have been many reports on its expression and function. However, limited information on ANO1 expression and function in the brain is still available. In this study, we tried to reexamine expression patterns of ANO1 in the mouse cerebellum and further characterize ANO1-expressing components by immunohistochemical analyses. Strong ANO1 immunoreactivity was observed as large puncta in the granule cell layer and weak to moderate immunoreactivities were observed as small puncta in the molecular and Purkinje cell layers. Double-label experiments revealed that ANO1 did not colocalize with cerebellar neuronal population markers, such as anti-calbindin and anti-NeuN, while it colocalized or intermingled with a presynaptic marker, anti-synaptophysin. These results demonstrate that ANO1 is mainly localized at presynaptic terminals in the cerebellum and involved in synaptic transmission and modulation in cerebellar information processing.

Key Words: Anoctamin 1, Calcium-activated chloride channel, Immunohistochemistry, Cerebellum

\section{INTRODUCTION}

Calcium $\left(\mathrm{Ca}^{2+}\right)$-activated chloride $\left(\mathrm{Cl}^{-}\right)$channels $(\mathrm{CaCCs})$ provide a chloride conductance activated by intracellular $\mathrm{Ca}^{2+}$. CaCCs fulfill essential functions in epithelial fluid transport, smooth muscle contraction, and sensory signal processing (Eggermont, 2004; Frings et al., 2000; Hartzell et al., 2005). Anoctamin 1 (ANO1) (also known as TMEM16A) and anoctamin 2 (ANO2, TMEM16B) have recently been identified as CaCCs (Caputo et al., 2008; Schroeder et al., 2008; Yang et al., 2008). In the nervous system, they are found in various types of sensory neurons and thus, considered to be involved in various sensory processing: olfaction (Billig et al., 2011; Stephan et al., 2009), vision (Jeon et al., 2013; Stöhr et al., 2009), hearing (Jeon et al., 2011; Zhang et al., 2015b), taste (Cherkashin et al., 2016), and pain and temperature (Cho et al., 2012; Lee et al., 2014). In addition, they are recently known to be expressed in hippocampus (Huang et al., 2012), thalamus (Ha et al., 2016), auditory brain stem nuclei (Cho et al., 2014), inferior olive (Zhang et al., 2017), and cerebellum (Neureither et al., 2017; Zhang et al., 2015a). Although their distribution and function has recently been unveiling in the brain, limited information is still available.

Cerebellum plays an important role in sensory-motor integration. In cerebellar circuitry, Purkinje cells that are the sole output neuron receive information from two afferent pathways: mossy fibers and climbing fibers. Mossy fibers originate from various brainstem nuclei and terminate as expanded boutons forming synaptic glomeruli where they give synaptic inputs onto granule cells. Afterwards, granule cell axons bifurcate into parallel fibers and make synapses onto Purkinje cell dendrites. Climbing fibers originate only one source, inferior olive. They entwine the Purkinje cell dendritic tree and make amazing numbers of synapses ( $\sim 500$ in each climbing fiber)

@ (T) This is an open-access article distributed under the terms of the Creative Commons Attribution Non-Commercial License (http://creativecommons.org/licenses/by-nc/4.0) which permits unrestricted noncommercial use, distribution, and reproduction in any medium, provided the original work is properly cited.

Copyrights @ 2018 by Korean Society of Microscopy 
(Llinás \& Walton, 1998). Classically, parallel fibers via mossy fibers and climbing fibers had been accepted to be primarily responsible for two distinctive electrophysiological activities of the Purkinje cell, simple spikes and complex spikes, respectively (Apps \& Garwicz, 2005; Bloedel \& Bracha, 2009). However, recent studies have propounded and proposed to revise both afferent pathway functions (Barmack \& Yakhnitsa, 2011), and thus, further studies on various ion channels eliciting electrophysiological activities are necessary.

Recently, ANO1 and ANO2 were found in the cerebellum (Cho et al., 2014; Neureither et al., 2017; Zhang et al., 2015a). In behavior and function, $\mathrm{ANO} 2^{-1-}$ mice showed impaired motor coordination and learning (Neureither et al., 2017; Zhang et al., 2017). However, there was a difference in cellular location of ANO2, that is, one group reported ANO2 expression in the Purkinje cell (Neureither et al., 2017; Zhang et al., 2015a), while the other group did its expression in climbing fibers (Zhang et al., 2017). There has been only one report to show ANO1 distribution in the cerebellum (Zhang et al., 2015a). Unlike ANO2, however, its physiological function and its significance in the cerebellum remain to be elusive. Here, we briefly report different findings about ANO1 distribution and localization in the mouse cerebellum, compared to those in a previous study (Zhang et al., 2015a).

\section{MATERIALS AND METHODS}

\section{Tissue Preparation}

Ten-week-old male C57BL/6 mice (Orient Bio, Korea) were euthanized with $15 \%$ chloral hydrate. Mice were transcardially perfused with saline and subsequently with $4 \%$ paraformaldehyde in $0.1 \mathrm{M}$ phosphate buffer (PB; pH 7.4). Brains were removed and post-fixed for 4 hours in the same fixative, then cryoprotected with $30 \%$ sucrose in $0.1 \mathrm{M} \mathrm{PB}$. For singleand double-labeling immunohistochemistry, brains were then embedded in Tissue-Tek (Sakura Finetechnical, Japan) and frozen with liquid nitrogen. The animals were treated according to the regulations of the Catholic Ethics Committee of the Catholic University of Korea, Seoul, which conform to the National Institute of Health (NIH) guidelines for the Care and Use of Laboratory Animals (NIH Publications No. 80-23) revised 1996.

\section{Antibodies}

Polyclonal antisera were raised in rabbits against a synthetic peptide with sequence of amino acids 451-466 (KDHPRAEYEARVLEKS) of mouse ANO1 (Ab Frontier, Korea). Specificity for this antiserum was previously tested by western blot and immunocytochemistry (Jeon et al., 2013; Yang et al., 2008) and also did in the knock-out controlled immunohistochemistry of the salivary gland (Romanenko et al., 2010). In order to determine the ANO1-labeled components, the fol- lowing antibodies for double-labeling experiments were used: mouse monoclonal anti-calbindin (Swant, Switzerland) as a marker for Purkinje cells, mouse monoclonal anti-neuronal nuclei (NeuN) (Chemicon, USA) as a marker for granule cells, and mouse monoclonal anti-synaptophysin (Sigma, USA) as a marker for presynaptic endings.

\section{Immunohistochemistry}

Coronal cryosections (50 $\mu \mathrm{m}$ thick) were processed for ANO1 immunohistochemistry. Free-floating sections were incubated in 10\% normal donkey serum and 1\% Triton X-100 in PB for 1 hour at room temperature, to block any nonspecific binding sites. They were then incubated with a polyclonal antibody against ANO1 (dilution 1:1,000) in PB containing 0.5\% Triton $\mathrm{X} 100$ for 1 day at $4^{\circ} \mathrm{C}$. The sections were washed in $\mathrm{PB}$ for 45 minutes ( $3 \times 15$ minutes), incubated in the presence of biotin-labeled goat anti-mouse IgG (Jackson Immuno Research, USA; dilution 1:100) for 2 hours, and then washed three times in $\mathrm{PB}$ for 45 minutes ( $3 \times 15$ minutes). The sections were then incubated in an avidin-biotin-peroxidase complex (ABC) solution (Vector Laboratories, USA) for 1 hour, washed in 0.1 $\mathrm{M}$ Tris buffer (TB; $\mathrm{pH} 7.6$ ), and then preincubated in $0.05 \%$ 3,3'-diaminobenzidine tetrahydrochloride (DAB) in TB for $10 \mathrm{~min}$, followed by incubation in the same solution containing $0.05 \%$ hydrogen peroxide $\left(\mathrm{H}_{2} \mathrm{O}_{2}\right)$ for an additional 10 minutes. The reaction was monitored using a low-power microscope and was stopped by replacing the $\mathrm{DAB}$ and $\mathrm{H}_{2} \mathrm{O}_{2}$ solution with TB.

For double-labeling, sections were incubated for 1 day in two primary antibody mixtures: a rabbit polyclonal antibody against ANO1 $(1: 1,000)$ and one of following mouse monoclonal antibodies, anti-calbindin (dilution, 1:3,000), antiNeuN (dilution, 1:2,000), and anti-synaptophysin (dilution, 1:2,000). The sections were rinsed for 30 minutes with PBS, and incubated in the presence of two secondary antibodies, donkey anti-rabbit conjugated with Cy3 (Jackson Immuno Research) and donkey anti-mouse conjugated with Alexa Fluor 488 (Molecular Probes, Eugene, OR) at a dilution of 1:200 in $\mathrm{PB}$ containing $0.5 \%$ Triton $\mathrm{X}-100$ in $\mathrm{PB}$ at $4^{\circ} \mathrm{C}$ for 2 to 4 hours. After rinsing several times in PBS, the fluorescent specimens were mounted with Vectashield mounting media (Vector Laboratories). Digital images $(1,024 \times 1,024$ pixels) were acquired by using a Zeiss LSM 510 Meta confocal microscope (Carl Zeiss, Germany) and processed using Photoshop (Adobe Systems, USA).

\section{RESULTS}

In consistent with our previous report that ANO1 was expressed in the mouse cerebellum at RNA and protein levels (Cho et al., 2014), ANO1 immunoreactivity was found in cerebellar cortex (Fig. 1). In light microscopy with a low magni- 

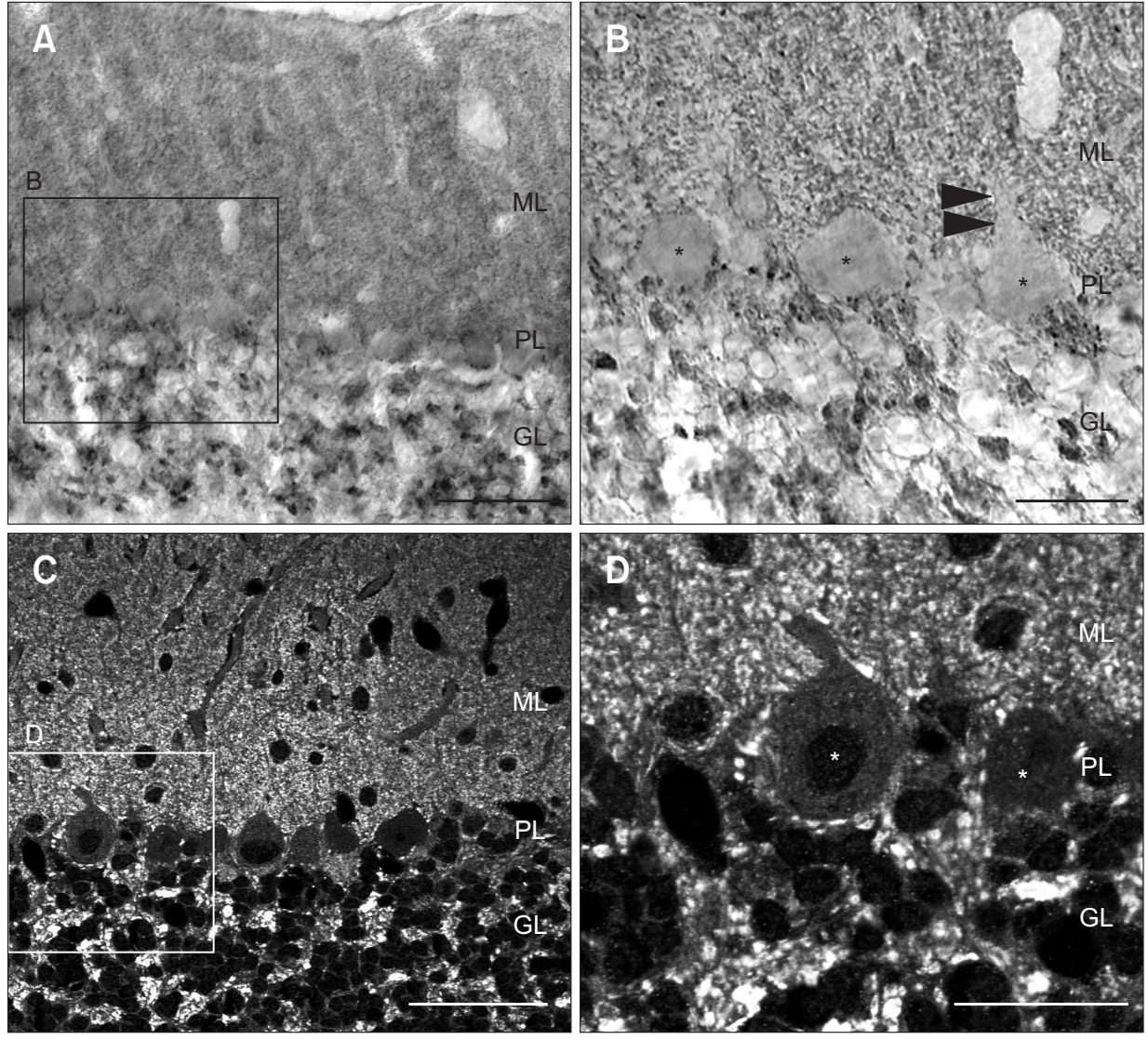

Fig. 1. Immunohistochemical localization of anoctamin 1 (ANO1) in the cerebellum. (A, B) Micrographs of a coronal section of the mouse cerebellum processed for immunohistochemistry with antiANO1. In Fig. 1A, weak immunoreactivity in molecular layer (ML) and strong immunoreactivity in granule cell layer (GL) was observed in low magnification view of cerebellum. In Fig. 1B, higher magnification view of the boxed area in Fig. 1A. Bodies (asterisks) of the Purkinje cells in the Purkinje cell layer (PL) and a primary apical dendrite (arrowheads) of a Purkinje cell did not show ANO1 immunoreactivity. (C, D) Confocal micrographs of a cerebellar coronal section processed for immunofluorescence with anti-ANO1. As shown in Fig. 1A and B, ANO1-labeled tiny puncta in the ML and larger puncta and patches in the GL are seen. Similar to Fig. 1B, Purkinje cell somata (asterisks) in the PL and a primary apical dendrite (arrowheads) did not show ANO1 immunoreactivity. Scale bars $=50 \mu \mathrm{m}(\mathrm{A}, \mathrm{C}), 20$ $\mu \mathrm{m}(\mathrm{B}, \mathrm{D})$. fication, weak ANO1 immunoreactivity was observed as tiny puncta in the molecular layer (ML) and the Purkinje cell layer (PL), while strong immunoreactivity was frequently observed as larger patches in the granule cell layer (GL) (Fig. 1A). At a higher magnification view, Purkinje cell bodies (asterisks) and a primary apical dendrite (arrowheads) appeared not to be or faintly labeled (Fig. 1B). The same results were reproduced in confocal microscopic observations (Fig. 1C and D). Compared to the results from light microscopy, numerous small labeled puncta were apparently seen in the ML and the GL.

Next, we tried to define cellular identity of ANO1-labeled components. First, to test whether ANO1-labeled components are related to the Purkinje cell components, we performed double-labeling with anti-ANO1 and anti-calbindin, a marker for Purkinje cell (Fig. 2A-C). ANO1 (red) do not colocalize with calbindin (green) in the ML, the PL, and the GL, (Fig. 2A-C), where dendrites, somata, and axons of Purkinje cells are labeled, respectively. These results demonstrate that ANO1 is not expressed in the Purkinje cells. To define the identity of the ANO1-labeled patches in the GL, we conducted doublelabeling with anti-ANO1 and anti-NeuN, which is known to specifically label granule cell somata in the cerebellum (Wolf et al., 1996) (Fig. 2D-F). As shown in Fig. 2D-F, ANO1-labeled patches (red) in the GL do not demonstrate NeuN immu- noreactivities, indicating that granule cells in the cerebellum do not express ANO1. Next, we examined whether ANO1labeled puncta in the ML and patches in the GL are presynaptic terminals or not, because ANO1 is localized at the presynaptic terminals in the retina (Jeon et al., 2013) and auditory brainstem (Cho et al., 2014). Double-labeling experiments with anti-ANO1 and synaptophysin, a presynaptic mossy fiber terminal marker (Leclerc et al., 1989), revealed that ANO1 generally colocalize with synaptophysin (Fig. 2G-I). Interestingly, ANO1 and synaptophysin immunoreactivities in the GL mostly appeared to intermingle. These results suggest that ANO1 is localized at synaptic varicosities of parallel fibers in the ML and mossy fibers and/or Golgi cell axons in the GL.

\section{DISCUSSION}

ANO1 is the firstly identified CaCC (Caputo et al., 2008; Schroeder et al., 2008; Yang et al., 2008), and among anoctamin family, it has been most extensively studied. It is known to be involved in $\mathrm{Cl}^{-}$secretion in various secretory epithelial tissues such as airways (Huang et al., 2009, 2012; Scudieri et al., 2012), salivary glands (Catalan et al., 2015; Romanenko et al., 2010; Yang et al., 2008), intestines (Namkung et al., 2011; Ousingsawat et al., 2009; Schreiber et al., 2015), and renal tu- 

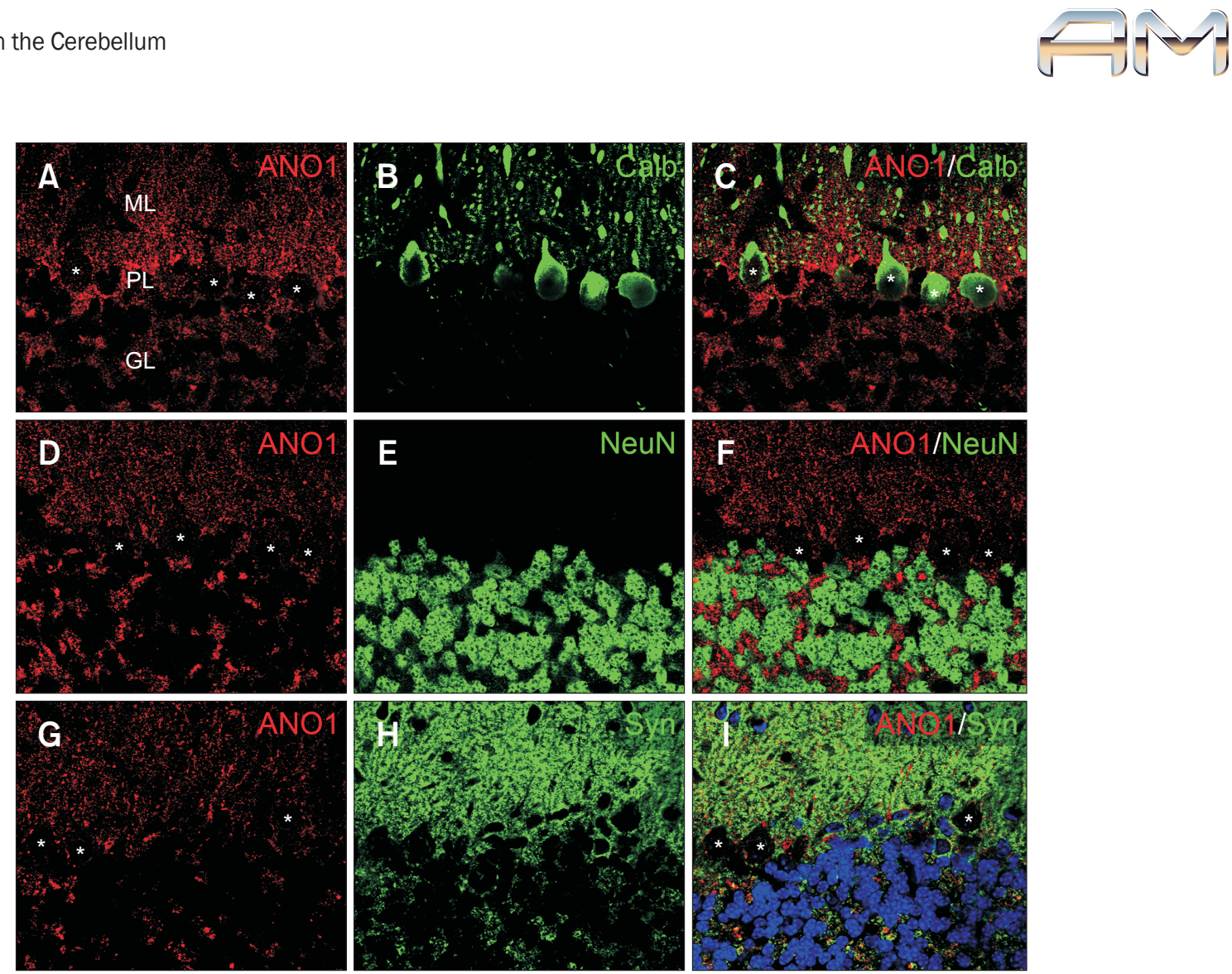

Fig. 2. Cellular identity of the anoctamin 1 (ANO1) in the cerebellum. Confocal micrographs taken from coronal sections of the mouse cerebellum processed for double-immunofluorescence. (A-C) Double-labeling with anti-ANO1 (A) and anti-calbindin (Calb) (B). In Fig. 2B, calbindin (green) is localized in the Purkinje cell dendrites in the molecular layer (ML) and somata in the Purkinje cell layer (PL). In the merged image (C) of Fig. 2A and B, calbindinlabeled Purkinje cell dendrites and somata (asterisks) did not show ANO1 immunoreactivities (red). (D-F) Double-labeling with anti-ANO1 (D) and antineuronal nuclei (NeuN) (E). In Fig. 2E, NeuN (green) is localized in the granule cell somata in the granule cell layer (GL). In the merged image (F) of Fig. 2D and E, NeuN-labeled granule cell somata did not show ANO1 immunoreactivities (red) in the GL. (G-I) Double-labeling experiments with anti-ANO1 (G) and anti-synaptophysin (Syn) (H). In Fig. 2H, synaptophysin immunoreactivities (green) are seen in many parallel fiber varicosities in the ML and some mossy fiber endings within the synaptic glomerulus in the GL. In the merged image (I) of Fig. 2G and H, synaptophysin-labeled endings and ANO1-labeled puncta and patches appear to be colocalized and/or intermingled in the ML and the GL. Asterisks indicate ANO1-unlabeled Purkinje cell somata in the PL. (A-I) Scale bars $=50 \mu \mathrm{m}$.

bules (Buchholz et al., 2014; Faria et al., 2014). ANO1 acts as a heat sensor (Cho \& Oh, 2013; Cho et al., 2012) and is involved in pain sensation (Lee et al., 2014; Liu et al., 2010; Takayama et al., 2015). ANO1 is also expressed in vascular and airway smooth muscles and involved in vascular tone (Bulley et al., 2012; Davis et al., 2012; Sun et al., 2012) and airway hypersensitivity (Huang et al., 2012), respectively. However, the expression and function of ANO1 in the central nervous system has not been studied extensively. There have only been reported that ANO1 is expressed in neurons in the retina (Jeon et al., 2013), and the auditory brainstem (Cho et al., 2014). Recently, the cerebellum is listed by Zhang et al. (2015a) and this study. In this study, ANO1 was observed in the parallel fiber varicosites of the ML and mossy fiber terminals and/or Golgi cell axons of the GL. These results are different from the previous report that ANO1 is expressed in inhibitory interneurons in the ML and the GL and Purkinje cell bodies in the PL (Zhang et al., 2015a). This discrepancy might be caused by the anti- bodies used. The antibody against ANO1 used in the study by Zhang et al. (2015a) labeled blood vessels and retinal pigment epithelium in the rat retina (Dauner et al., 2013), while our ANO1 antibody labeled presynaptic terminals of photoreceptors, bipolar and amacrine terminals in the mouse retina (Jeon et al., 2013). Specificity for the antibody used in this study was previously tested by western blot and immunocytochemistry (Jeon et al., 2013; Yang et al., 2008) and also did in the knockout controlled immunohistochemistry of the salivary gland (Romanenko et al., 2010). In addition, in our previous study in the mouse retina, we successfully identified $\mathrm{Ca}^{2+}$-activated $\mathrm{Cl}^{-}$current $\left(\mathrm{I}_{\mathrm{Cl}(\mathrm{Ca})}\right)$ in the rod bipolar cells in which ANO1 is expressed, and $\mathrm{T}_{16 \mathrm{~A}_{\text {inh }}}$-A01, a specific ANO1 inhibitor inhibited the $\mathrm{I}_{\mathrm{Cl}(\mathrm{Ca})}$ (Jeon et al., 2013). These indicate that ANO1 antibody used in this study is reliable one.

Recently, expression and function of ANO2 in the cerebellum were reported by two research groups. One group reported that ANO2 was expressed in postsynaptic Purkinje cell 
dendrites and demonstrated that it modulated GABAergic synapse in a $\mathrm{Ca}^{2+}$-dependent manner (Zhang et al., 2015a). Afterwards, they demonstrated that $\mathrm{ANO}^{-1-}$ mice showed impaired motor coordination and learning (Neureither et al., 2017). The other group observed the similar cerebellar impairment in $\mathrm{ANO}_{2}^{-l-}$ mice. However, based on the findings that mCherry signal expressed in ANO2-expression cells was detected in climbing fibers synapsing onto the Purkinje cells in the cerebellum and ANO2 was involved in repolarization of the inferior olivary neurons, they differently interpreted, that is, the behavioral deficits of $\mathrm{ANO}^{-1-}$ mice were caused by the absence of ANO2 in inferior olivary neurons (Zhang et al., 2017). In addition, in this study, our findings on ANO1 localization in the cerebellum are different from ones by Zhang et al. (2015a). Taken together, further studies including immunoelectron microscopy with immuno-gold are needed to conclude or reconcile above discrepancies.

One explanation for reconciliation might be possible, albeit unlikely. In the present study, not a few ANO1-immunoreactive puncta and/or patches in the cerebellum are intermingled rather than colocalized with synaptophysin immunoreactivities, particularly in the GL. Based on a previous report that synaptophysin is labeled in many parallel fiber varicosities and mossy fiber endings (Leclerc et al., 1989), these results may suggest that ANO1 may be localized in Golgi cell axons in the GL, and basket/stellate cell processes in the ML. Another explanation might be that ANO1 in synaptic glomeruli in the GL is localized in some specific mossy fiber rosettes, not all mossy fibers, because mossy fibers are originated from various brainstem regions, including pontine nuclei, vestibular nuclei, and so on (Llinás \& Walton, 1998).

Lastly, we propose that ANO1 may contribute to transmit information at high-frequency at mossy fiber-granule cell synapse by shortening of the presynaptic action potential duration. Cerebellar mossy fiber synapse is known to have the highest firing frequencies (Delvendahl \& Hallermann, 2016). Several presynaptic mechanisms including short duration of the presynaptic action potential have been proposed for this high-frequency transmission. In our previous study in the retina, we demonstrated that presynaptically localized ANO1 could shorten $\mathrm{Ca}^{2+}$ spike-like depolarizations, which resemble $\mathrm{Na}^{+}$action potentials (Jeon et al., 2013). Interestingly, ANO1 is localized in large axosomatic endings called the calyces of Held and endbulbs of Held (Cho et al., 2014), in which highfrequency synaptic transmission through fast, precise, and resilient action potential firing (Borst et al., 1995; Forsythe \& Barnes-Davies, 1993). These findings could support our proposal for ANO1 function in the mossy fiber rosettes.

\section{CONCLUSIONS}

In this study, our results clearly showed presynaptic ANO1 localization in the mouse cerebellum. The results suggest potential roles in synaptic transmission and modulation in cerebellar information processing, particularly high-frequency transmission at mossy fiber-granule cell synapse.

\section{CONFLICT OF INTEREST}

No potential conflict of interest relevant to this article was reported.

\section{ACKNOWLEDGMENTS}

This study has been supported by National Research Foundation of Korea (NRF) funded by the Ministry of Science and ICT (NRF-2017R1A2B2005309).

\section{REFERENCES}

Apps R and Garwicz M (2005) Anatomical and physiological foundations of cerebellar information processing. Nat. Rev. Neurosci. 6, 297-311.

Barmack N H and Yakhnitsa V (2011) Topsy turvy: functions of climbing and mossy fibers in the vestibulo-cerebellum. Neuroscientist 17, 221-236.

Billig G M, Pál B, Fidzinski P, and Jentsch T J (2011) $\mathrm{Ca}^{2+}$-activated Cl currents are dispensable for olfaction. Nat. Neurosci. 14, 763-769.

Bloedel J R and Bracha V (2009) Cerebellar functions. In: Encyclopedic Reference of Neuroscience, eds. Binder M D, Hirokawa N, Windhorst U, pp. 667-671, (Springer-Verlag, Heidelberg).

Borst J G and Sakmann B (1995) Pre- and postsynaptic whole-cell recordings in the medial nucleus of the trapezoid body of the rat. J. Physiol. 489, 825-840.
Buchholz B, Faria D, Schley G, Schreiber R, Eckardt K U, and Kunzelmann K (2014) Anoctamin 1 induces calcium-activated chloride secretion and proliferation of renal cyst-forming epithelial cells. Kidney Int. 85, 1058-1067.

Bulley S, Neeb Z P, Burris S K, Bannister J P, Thomas-Gatewood C M, Jangsangthong W, and Jaggar J H (2012) TMEM16A/ANO1 channels contribute to the myogenic response in cerebral arteries. Circ. Res. 111, $1027-1036$.

Caputo A, Caci E, Ferrera L, Pedemonte N, Barsanti C, Sondo E, Pfeffer U, Ravazzolo R, Zegarra-Moran O, and Galietta L J (2008) TMEM16A, a membrane protein associated with calcium-dependent chloride channel activity. Science 322, 590-594.

Catalan M A, Kondo Y, Pena-Munzenmayer G, Jaramillo Y, Liu F, Choi S, Crandall E, Borok Z, Flodby P, Shull G E, and Melvin J E (2015) A fluid 
secretion pathway unmasked by acinar-specific Tmem16A gene ablation in the adult mouse salivary gland. Proc. Natl. Acad. Sci. U. S. A. 112, 2263-2268.

Cherkashin A P, Kolesnikova A S, Tarasov M V, Romanov R A, Rogachevskaja O A, Bystrova M F, and Kolesnikov S S (2016) Expression of calcium-activated chloride channels Ano1 and Ano2 in mouse taste cells. Pflugers Arch. 468, 305-319.

Cho $\mathrm{H}$ and Oh U (2013) Anoctamin 1 mediates thermal pain as a heat sensor. Curr. Neuropharmacol. 11, 641-651.

Cho H, Yang Y D, Lee J, Lee B, Kim T, Jang Y, Back S K, Na H S, Harfe B D, Wang F, Raouf R, Wood J N, and Oh U (2012) The calcium-activated chloride channel anoctamin 1 acts as a heat sensor in nociceptive neurons. Nat. Neurosci. 15, 1015-1021.

Cho S J, Jeon J H, Chun D I, Yeo S W, and Kim I B (2014) Anoctamin 1 expression in the mouse auditory brainstem. Cell Tissue Res. 357, 563-569.

Dauner K, Möbus C, Frings S, and Möhrlen F (2013) Targeted expression of anoctamin calcium-activated chloride channels in rod photoreceptor terminals of the rodent retina. Invest. Ophthalmol. Vis. Sci. 54, 3126-3136.

Davis A J, Shi J, Pritchard H A, Chadha P S, Leblanc N, Vasilikostas G, Yao Z, Verkman A S, Albert A P, and Greenwood I A (2012) Potent vasorelaxant activity of the TMEM16A inhibitor T16A(inh)-A01. Br. J. Pharmacol. 168, 773-784.

Delvendahl I and Hallermann S (2016) The cerebellar mossy fiber synapse as a model for high-frequency transmission in the mammalian CNS. Trends Neurosci. 39, 722-737.

Eggermont J (2004) Calcium-activated chloride channels: (un)known, (un) loved? Proc. Am. Thorac. Soc. 1, 22-27.

Faria D, Rock J R, Romao A M, Schweda F, Bandulik S, Witzgall R, Schlatter E, Heitzmann D, Pavenstadt H, Herrmann E, Kunzelmann K, and Schreiber R (2014) The calcium-activated chloride channel anoctamin 1 contributes to the regulation of renal function. Kidney Int. $\mathbf{8 5}$, 1369-1381.

Frings S, Reuter D, and Kleene S J (2000) Neuronal $\mathrm{Ca}^{2+}$-activated $\mathrm{Cl}^{-}$ channels: homing in on an elusive channel species. Prog. Neurobiol. 60, 247-289.

Forsythe I D and Barnes-Davies M (1993) The binaural auditory pathway: membrane currents limiting multiple action potential generation in the rat medial nucleus of the trapezoid body. Proc. Biol. Sci. 251, 143-150.

Ha G E, Lee J, Kwak H, Song K, Kwon J, Jung S Y, Hong J, Chang G E, Hwang E M, Shin H S, Lee C J, and Cheong E (2016) The $\mathrm{Ca}^{2+}$ activated chloride channel anoctamin-2 mediates spike-frequency adaptation and regulates sensory transmission in thalamocortical neurons. Nat. Commun. 7, 13791.

Hartzell C, Putzier I, and Arreola J (2005) Calcium-activated chloride channels. Annu. Rev. Physiol. 67, 719-758.

Huang F, Rock J R, Harfe B D, Cheng T, Huang X, Jan Y N, and Jan L Y (2009) Studies on expression and function of the TMEM16A calciumactivated chloride channel. Proc. Natl. Acad. Sci. U. S. A. 106, 2141321418.

Huang F, Zhang H, Wu M, Yang H, Kudo M, Peters CJ, Woodruff P G, Solberg O D, Donne M L, Huang X, Sheppard D, Fahy J V, Wolters P J, Hogan BL, Finkbeiner W E, Li M, Jan Y N, Jan L Y, and Rock J R (2012) Calcium-activated chloride channel TMEM16A modulates mucin secretion and airway smooth muscle contraction. Proc. Natl. Acad. Sci. U. S. A. 109, 16354-16359.
Huang W C, Xiao S, Huang F, Harfe B D, Jan Y N, Jan L Y (2012) Calciumactivated chloride channels (CaCCs) regulate action potential and synaptic response in hippocampal neurons. Neuron 74, 179-192.

Jeon J H, Paik S S, Chun M H, Oh U, and Kim I B (2013) Presynaptic localization and possible function of calcium-activated chloride channel anoctamin 1 in the mammalian retina. PLoS One 8, e67989.

Jeon J H, Park J W, Lee J W, Jeong S W, Yeo S W, and Kim I B (2011) Expression and immunohistochemical localization of TMEM16A/ anoctamin 1, a calcium-activated chloride channel in the mouse cochlea. Cell Tissue Res. 345, 223-230.

Leclerc N, Beesley P W, Brown I, Colonnier M, Gurd J W, Paladino T, and Hawkes R (1989) Synaptophysin expression during synaptogenesis in the rat cerebellar cortex. J. Comp. Neurol. 280, 197-212.

Lee B, Cho H, Jung J, Yang Y D, Yang D J, and Oh U (2014) Anoctamin 1 contributes to inflammatory and nerve-injury induced hypersensitivity. Mol. Pain 10, 5.

Liu B, Linley J E, Du X, Zhang X, Ooi L, Zhang H, and Gamper N (2010) The acute nociceptive signals induced by bradykinin in rat sensory neurons are mediated by inhibition of M-type $\mathrm{K}+$ channels and activation of Ca2+-activated Cl- channels. J. Clin. Invest. 120, 1240-1252.

Llinás R R and Walton K D (1998) Cerebellum. In: The Synaptic Organization of the Brain, ed. Shepherd G M, pp. 255-288, (Oxford University Press, Oxford).

Namkung W, Phuan P W, and Verkman A S (2011) TMEM16A inhibitors reveal TMEM16A as a minor component of calcium-activated chloride channel conductance in airway and intestinal epithelial cells. $J$. Biol. Chem. 286, 2365-2374.

Neureither F, Ziegler K, Pitzer C, Frings S, and Möhrlen F (2017) Impaired motor coordination and learning in mice lacking anoctamin 2 calcium-gated chloride channels. Cerebellum 16, 929-937.

Ousingsawat J, Martins J R, Schreiber R, Rock J R, Harfe B D, and Kunzelmann K (2009) Loss of TMEM16A causes a defect in epithelial Ca2+-dependent chloride transport. J. Biol. Chem. 284, 2869828703.

Romanenko V G, Catalan M A, Brown D A, Putzier I, Hartzell H C, Marmorstein A D, Gonzalez-Begne M, Rock J R, Harfe B D, and Melvin J E (2010) Tmem16A encodes the Ca2+-activated Cl- channel in mouse submandibular salivary gland acinar cells. J. Biol. Chem. 285, 12990-13001.

Schreiber R, Faria D, Skryabin B V, Wanitchakool P, Rock J R, and Kunzelmann K (2015) Anoctamins support calcium-dependent chloride secretion by facilitating calcium signaling in adult mouse intestine. Pflugers Arch. 467, 1203-1213.

Schroeder B C, Cheng Y, Jan Y N, and Jan L Y (2008) Expression cloning of TMEM16A as a calcium-activated chloride channel subunit. Cell 134, 1019-1029.

Scudieri P, Caci E, Bruno S, Ferrera L, Schiavon M, Sondo E, Tomati V, Gianotti A, Zegarra-Moran O, Pedemonte N, Rea F, Ravazzolo R, and Galietta L J (2012) Association of TMEM16A chloride channel overexpression with airway goblet cell metaplasia. J. Physiol. 590, 61416155.

Stephan A B, Shum E Y, Hirsh S, Cygnar K D, Reisert J, and Zhao H (2009) ANO2 is the cilial calcium-activated chloride channel that may mediate olfactory amplification. Proc. Natl. Acad. Sci. U. S. A. 106, 1177611781.

Stöhr H, Heisig J B, Benz P M, Schöberl S, Milenkovic V M, Strauss O, Aartsen W M, Wijnholds J, Weber B H, and Schulz H L (2009) TMEM16B, a novel protein with calcium-dependent chloride channel 
activity, associates with a presynaptic protein complex in photoreceptor terminals. J. Neurosci. 29, 6809-6818.

Sun H, Xia Y, Paudel O, Yang X R, and Sham J S (2012) Chronic hypoxiainduced upregulation of $\mathrm{Ca} 2+$-activated $\mathrm{Cl}$ - channel in pulmonary arterial myocytes: a mechanism contributing to enhanced vasoreactivity. J. Physiol. 590, 3507-3521.

Takayama Y, Uta D, Furue H, and Tominaga M (2015) Pain-enhancing mechanism through interaction between TRPV1 and anoctamin 1 in sensory neurons. Proc. Natl. Acad. Sci. U. S. A. 112, 5213-5218.

Wolf H K, Buslei R, Schmidt-Kastner R, Schmidt-Kastner P K, Pietsch T, Wiestler O D, and Blümcke I (1996) NeuN: a useful neuronal marker for diagnostic histopathology. J. Histochem. Cytochem. 44, 11671171.

Yang Y D, Cho H, Koo J Y, Tak M H, Cho Y, Shim W S, Park S P, Lee J, Lee
B, Kim B M, Raouf R, Shin Y K, and Oh U (2008) TMEM16A confers receptor-activated calcium-dependent chloride conductance. Nature 455, $1210-1215$.

Zhang W, Schmelzeisen S, Parthier D, Frings S, and Möhrlen F (2015a) Anoctamin calcium-activated chloride channels may modulate inhibitory transmission in the cerebellar cortex. PLoS One 10, e0142160.

Zhang X D, Lee J H, Lv P, Chen W C, Kim H J, Wei D, Wang W, Sihn CR, Doyle K J, Rock J R, Chiamvimonvat N, and Yamoah E N (2015b) Etiology of distinct membrane excitability in pre- and posthearing auditory neurons relies on activity of $\mathrm{Cl}$ - channel TMEM16A. Proc. Natl. Acad. Sci. U. S. A. 112, 2575-2580.

Zhang Y, Zhang Z, Xiao S, Tien J, Le S, Le T, Jan L Y, and Yang H (2017) Inferior olivary TMEM16B mediates cerebellar motor learning. Neuron 95, 1103-1111. 